\title{
REVIEW
}

\section{Recreational drug misuse: issues for the cardiologist}

\author{
A Ghuran, J Nolan
}

Alcohol and tobacco are the two legally available recreational drugs that are commonly misused in the United Kingdom, and their adverse effects on cardiovascular function have been extensively documented. In addition, many illegal recreational drugs are freely available, and their use has reached epidemic proportions in British society. Almost one in four people have used illegal drugs at some time during their life, with a proportionately higher rate of use in younger age groups. A 1996 Home Office survey showed that more than three million British people use an illegal drug every month. In addition to the problems posed by self administration of these agents, massive overdose can occur in individuals ("body packers") who attempt to smuggle drugs by ingesting packets which rupture accidentally in the gastrointestinal tract. It is therefore inevitable that doctors will have to manage the ill effects associated with these drugs. Many of these agents have profound effects on the heart and circulation that are responsible for a large proportion of drug related deaths. Our purpose in this article is to review the cardiovascular side effects of the commonly misused non-prescription illegal recreational drugs, and provide guidelines for the management of these potentially lethal cardiovascular complications, based on the best available evidence.

\section{Cocaine, amphetamine, "ecstasy"}

Cocaine, amphetamine, and ecstasy have similar adverse effects on the cardiovascular system, predominantly related to activation of the sympathetic nervous system. Cocaine and its free base form "crack" are absorbed from all mucous membranes of the body, and can be smoked, inhaled, or injected. Cocaine is an indirectly acting sympathomimetic drug that inhibits the reuptake of noradrenaline (norepinephrine) and dopamine at sympathetic nerve terminals, and can also act through central pathways to release noradrenaline from the adrenal medulla. ${ }^{1}$ Circulating catecholamine concentrations can be raised as much as fivefold in cocaine users. ${ }^{2}$ At high dose levels, cocaine can impair myocyte electrical activity and contractility by blocking fast sodium and potassium channels and inhibiting calcium entry into myocytes. ${ }^{2}$ Cocaine has a short serum half life of approximately 30-80 min- utes, with $90 \%$ being metabolised and excreted in the urine over a two week period, providing a means of retrospectively diagnosing recent ingestion. ${ }^{3}$ Some of the metabolites of cocaine (particularly cocaethylene, formed when cocaine is taken with alcohol) are more cardiotoxic than the parent compound. ${ }^{4}$ Cannabis can potentiate the toxic effects of cocaine by increasing plasma concentrations of the drug. ${ }^{5}$

Amphetamine can be ingested orally, inhaled, or less commonly injected intravenously. It is readily absorbed from both the nasal mucosa and the gastrointestinal tract, and freely penetrates the blood-brain barrier. Metabolism is highly variable, and up to $30 \%$ of the parent compound can be excreted unchanged in the urine. The plasma half life varies from as little as five hours to as much as 20-30 hours depending on urine flow and $\mathrm{pH}$, with drug elimination being increased in acidic urine. Amphetamine or its metabolites can be detected in the urine for several days after ingestion, and excretion is prolonged after administration of large doses or in the presence of alkaline urine (some misusers deliberately take bicarbonate at the same time to delay excretion and enhance the drug effect).

Ecstasy is a derivative of amphetamine and has similar effects. The effects begin approximately 20 minutes after ingestion and generally last up to six hours, although larger doses can have an effect for up to 48 hours. Ecstasy is metabolised by the liver and excreted by the kidney.

Both amphetamine and ecstasy produce indirect sympathetic activation by releasing noradrenaline (norepinephrine), dopamine, and serotonin from terminals in the central and autonomic nervous systems. In contrast to cocaine, amphetamine can inhibit the enzyme monoamine oxidase and lacks the local anaesthetic effect of inhibiting fast sodium channels. ${ }^{6}$ Amphetamine toxicity is potentiated when it is taken with alcohol ${ }^{7}$.

Drug induced sympathetic activation leads to varying degrees of tachycardia, vasoconstriction, unpredictable blood pressure effects, and arrhythmias, depending on the dose given and the presence or absence of coexisting cardiovascular disease. Hypertension is common, but severe hypotension (related to paradoxical central sympathetic suppression, a late state of catecholamine depletion, or ventricular impair- 
ment induced by ischaemia or mechanical complications) can also occur. ${ }^{289}$ Myocardial ischaemia and infarction can be produced by various mechanisms. Diffuse or local coronary artery spasm can be induced in normal or atherosclerotic coronary arteries. ${ }^{10-15}$ In vitro studies suggest that cocaine produces a procoagulant effect by decreasing concentrations of protein $\mathrm{C}$ and antithrombin 3 , activating platelets, and potentiating thromboxane production, facilitating thrombotic coronary occlusion. ${ }^{216}$ The haemodynamic changes associated with sympathetic stimulation increase myocardial oxygen demand. Chronic use of cocaine and amphetamine can cause repetitive episodes of coronary spasm and paroxysms of hypertension, which may result in endothelial damage, coronary artery dissection, and subsequent acceleration of atherosclerosis. ${ }^{11}{ }^{13} \mathrm{~A}$ necrotising vasculitis involving medium and small arteries in most organs can lead to widespread ischaemia in some amphetamine misusers. ${ }^{12} 18$

Prolonged administration of these sympathomimetic agents may be associated with an irreversible dilated cardiomyopathy. ${ }^{19-21}$ Possible aetiological mechanisms are related to subendocardial ischaemia and fibrosis (characterised by contraction band necrosis) and myocyte necrosis produced by exposure to excessive catecholamine concentrations or repeated episodes of myocarditis. Myocardial cellular injury can also occur in association with exposure to infectious agents or heavy metals, such as manganese, that contaminate street preparations of cocaine. Noncardiogenic pulmonary oedema (NCPO) and pulmonary hypertension can also occur with cocaine and amphetamine misuse. The precise mechanism underlying this is uncertain, but a direct toxic effect on pulmonary vessels could be involved. ${ }^{6}{ }^{19}$ Another possible mechanism of NCPO is a drug induced change in central autonomic regulatory activity, producing an indirect effect on pulmonary vascular function. ${ }^{6}{ }^{19}$

Administration of sympathomimetic agents is occasionally associated with important mechanical complications. ${ }^{22}$ Paroxysmal increases in blood pressure can lead to aortic dissection or valve trauma which increases the risk of endocarditis. When endocarditis occurs, it mainly affects the aortic and mitral valves, ${ }^{2}$ as it is the left sided heart valves that are subjected to the maximum degree of hypertension related stress and damage. Endocarditis is often associated with unusual organisms such as candida, pseudomonas, or klebsiella, and frequently has an aggressive clinical course with severe valvar destruction, abscess formation, and a requirement for surgical intervention. Some cocaine misusers practice drug inhalation in association with a forced Valsalva manoeuvre (which enhances the drug effect), and this may occasionally result in symptomatic pneumothorax or pneumopericardium.

The adverse cardiovascular changes and sympathetic stimulation associated with administration of these compounds can precipitate a wide and unpredictable range of supraventricular and potentially lethal ventricular tachyarrhythmias. The presence of myocardial ischaemia, fibrotic contraction bands, and left ventricular hypertrophy can act as a substrate for re-entrant arrhythmias. Owing to its class 1 antiarrhythmic effects, cocaine can impair cardiac conduction, inducing a wide range of bradyarrhythmias including sinus arrest and atrioventricular block. ${ }^{23} 24$ Sudden cardiovascular collapse may occur as a result of myocardial ischaemia and infarction, arrhythmias, acute heart failure, or mechanical complications.

Similar principles apply to the management of the cardiovascular complications associated with these three recreational drugs, although the duration of treatment will vary depending on the half life of the agent taken. Benzodiazepines attenuate the cardiac and central nervous system toxicity of these drugs and should be given in sedative dosages. ${ }^{25}$ Short lived depression of cardiac function may be severe, and require a period of mechanical cardiac and ventilatory support. ${ }^{25}$ In the treatment of hypertension, $\beta$ blockers should be avoided as they may be associated with unopposed $\alpha$ receptor mediated vasoconstriction, which can result in a sudden and severe increase in blood pressure and coronary artery vasoconstriction. The combined $\alpha$ and $\beta$ blocking drug labetalol is theoretically preferable to pure $\beta$ blockers. ${ }^{26}$ The clinical effects of this drug are, however, unpredictable as it has only relatively weak $\alpha$ blocking properties and may therefore exacerbate hypertension. ${ }^{25}$ In body packers suffering from overdose after rupture of ingested packets of cocaine, calcium antagonists may accelerate gastrointestinal drug absorption by inducing splanchnic vasodilatation. Additionally, cocaine has a complex and highly variable effect on myocyte calcium metabolism, producing an unpredictable clinical response to calcium antagonists. ${ }^{27-30}$ Because of these concerns, it may be preferable to avoid giving calcium antagonists to patients suspected of cocaine misuse. Hypertension can be safely managed with either an $\alpha$ blocker such as phentolamine or direct acting vasodilators such as nitrates, hydralazine, or nitroprusside. ${ }^{25}$ When hypertensive crises lead to the mechanical complications of aortic dissection or acute valve rupture, emergency corrective surgery may be required.

Myocardial ischaemia induced by these drugs should be treated initially with oxygen, aspirin, and benzodiazepines. If there is continuing ischaemia then the use of additional vasodilators such as nitrates or phentolamine to reverse residual coronary spasm may be necessary. ${ }^{25}$ If there is persistent ST segment elevation on the ECG, reperfusion should be considered. Providing that blood pressure is controlled and there is no evidence of haemorrhagic complications, thrombolytic treatment is safe in myocardial infarction associated with cocaine and amphetamine misuse. ${ }^{15}{ }^{31}$ Primary angioplasty should be considered if thrombolysis is contraindicated because of bleeding problems. Overall mortality from myocardial infarction related to these compounds is low, 
reflecting the age and general health of the majority of patients. ${ }^{32}$

Published evidence relating to the management of arrhythmias induced by these agents is limited, but some general principles can be used to guide treatment. In the management of short lived arrhythmias, drug treatment should be avoided if possible, as antiarrhythmic agents and cocaine may have additive effects on myocyte electrolyte transport and electrophysiological properties, leading to synergistic depression of contractile function or proarrhythmia. Many arrhythmias will terminate spontaneously as the drug is metabolised and cardiac function returns to normal. Supraventricular or ventricular tachyarrhythmias associated with haemodynamic compromise require urgent DC cardioversion. For sustained haemodynamically tolerated supraventricular tachyarrhythmias associated with atrioventricular (AV) nodal re-entry, adenosine is safe and free of major side effects. In the presence of a hyperadrenergic state, the short lived dromotropic effects of adenosine may be a disadvantage, allowing rapid reinduction of the tachyarrhythmia. If adenosine is unsuccessful, the arrhythmia rapidly returns, or a mechanism other than AV nodal re-entry is operating, administration of an $\alpha$ antagonist and a $\beta$ blocker in combination is likely to be both safe and effective. Although atropine has been used to treat bradyarrhythmias, ${ }^{24}$ its effects may be attenuated in the presence of a hyperadrenergic state, and temporary cardiac pacing may be necessary. ${ }^{23}$ In the management of sustained ventricular tachyarrhythmias lignocaine (lidocaine) and magnesium have an acceptable safety and efficacy profile (despite theoretical concerns relating to the shared class I effects of cocaine and lignocaine). ${ }^{27} 33$ There is currently no reliable information on the safety and efficacy of other antiarrhythmic drugs. In animal studies of cocaine, the administration of sodium bicarbonate has demonstrable beneficial effects on myocardial electrical stability. ${ }^{34}$ However, this may occur at the expense of inducing paradoxical intracellular acidosis or adverse systemic metabolic changes, leading to detrimental effects on myocardial function. Similarly, in severe cases of amphetamine overdose, a forced acid diuresis may be successful in rapidly clearing amphetamine from the blood and limiting toxicity, ${ }^{35}$ but major detrimental metabolic changes in acid-base balance can be induced. These treatments require intensive and expert monitoring and should only be performed by clinicians with previous experience of metabolically invasive therapy.

\section{Lysergic acid diethylamide (LSD) and psilocybin ("magic mushrooms")}

The hallucinogenic agents lysergic acid diethylamide (LSD) and psilocybin ("magic mushrooms") are used recreationally by large numbers of individuals in developed countries. Psilocybin is derived from a common mushroom species which grows wild throughout the UK, whereas LSD (which was also originally extracted from a fungus) is now usually manu- factured synthetically. These drugs are structurally related and have similar physiological, pharmacological, and clinical effects. Both drugs are usually ingested orally, with LSD being 100 times more potent than psilocybin. Street mushrooms are often adulterated with LSD. Both drugs are indole derivatives and chemically resemble serotonin. Their mechanisms of action are complex and include agonist, partial agonist, and antagonist effects at various serotonin, dopaminergic, and adrenergic receptors. ${ }^{36}{ }^{37}$ The adrenergic effects are usually mild and do not produce the profound sympathetic storms that can occur after taking cocaine, amphetamine, or ecstasy. Following ingestion, the central nervous system effects begin within 15 to 45 minutes and usually last for four to six hours. LSD is metabolised by the liver, has a plasma half life of 100 minutes, ${ }^{38}$ and can be detected in plasma or urine for up to three days. Animal studies have shown that $65 \%$ of psilocybin is excreted in the urine and $15-20 \%$ in bile and faeces; most of the excretion occurs within the first eight hours, with small amounts being excreted for up to seven days. ${ }^{37}$

These drugs are well known for their powerful mind altering properties, causing feelings of euphoria, anxiety, paranoia, and visual and auditory hallucinations which can occur days, months, or even up to five years after the last use. ${ }^{38}$ Symptoms that correspond to general sympathetic arousal include dilated pupils, tachycardia, hypertension, and hyperreflexia. ${ }^{38}$ Abnormalities of serotonin induced platelet aggregation may result in abnormal clotting and poor clot retraction. ${ }^{39}$ Cardiovascular complications are rarely serious, although occasional instances of supraventricular tachyarrhythmias and myocardial infarction have been reported. ${ }^{40}$ Although the exact mechanism is not known, alteration of serotonin induced platelet function and sympathetically induced arterial vasospasm may contribute to these cardiovascular complications.

The management of LSD and psilocybin intoxication is usually supportive, as the majority of symptoms resolve within 12 hours. ${ }^{41}$ Agitated patients should be sedated with a benzodiazepine. The use of neuroleptic agents should be avoided as they can intensify toxic effects. ${ }^{38}$ Supraventricular tachyarrhythmias can be treated with adenosine or verapamil. ${ }^{40}$ Apart from benzodiazepines, pharmacological intervention for mild to moderate hypertension is usually not required. Treatment for dangerously high blood pressure and myocardial ischaemia should follow the same general principles described for cocaine, amphetamine, and ecstasy intoxication.

\section{Narcotic analgesics}

Narcotic analgesics are commonly injected, smoked, or ingested orally. The most commonly misused narcotic analgesics are heroin and morphine. Heroin (diacetylmorphine) is a semisynthetic analogue of morphine which is slowly metabolised to the parent compound. Heroin is more lipid soluble than morphine and therefore acts more rapidly. Morphine has 
a plasma half life of two to three hours. It undergoes rapid hepatic metabolism and the metabolites are excreted in the urine. The duration of renal excretion is highly variable and is affected by the dose given, the precise chemical composition of street preparations, the user's previous drug habits, and individual variations in renal and hepatic function. In general, metabolites can be detected for up to 48 hours in occasional users, and for several days in chronic misusers. Narcotic analgesics act directly on the vasomotor centre to increase parasympathetic activity, reduce sympathetic activity, and release histamine from mast cells. These effects combine to produce bradycardia and hypotension. Drug induced bradycardia along with enhanced automaticity can precipitate an increase in ectopic activity, atrial fibrillation, idioventricular rhythm, or potentially lethal ventricular tachyarrhythmias. ${ }^{42}$ Some narcotic analgesics (such as dextropropoxyphene, a constituent of coproxamol) have additional sodium channel blocking effects, which further contribute to the proarrhythmic ${ }^{43}$ and myocardial depressant effects, ${ }^{44}$ leading to acute left ventricular dysfunction and pulmonary oedema.

Overdose of narcotic analgesics can cause acute NCPO, the onset of which may be delayed for up to 24 hours after admission. ${ }^{45-47}$ This may be related to an anaphylactic reaction to the drug, to an increase in pulmonary capillary hydrostatic pressure resulting from pulmonary vasoconstriction induced by hypoxia, or to disruption of alveolar capillary membrane integrity. The administration of narcotic analgesic receptor blockers may precipitate the development of NCPO by reversing opioid induced vasodilatation and venous pooling, thereby increasing venous return to the lungs.

Apart from the well described central nervous system and respiratory depressant effects, there may also be profound cardiovascular collapse or arrhythmias after narcotic analgesic overdose. Evidence of intravenous drug administration may be absent in some users who smoke the drug, and a high index of suspicion based on associated features may allow a retrospective diagnosis to be established by urine toxicology. When intravenous narcotic misusers present chronically with bacterial endocarditis, it usually affects the right sided heart valves and may be associated with pulmonary abscess formation.

The initial management of narcotic analgesic overdose requires the establishment of a stable airway and ventilatory support if necessary. In patients who are haemodynamically stable, it may be preferable to avoid the administration of narcotic analgesic receptor antagonists, because of the risk of precipitating NCPO. In the presence of severe hypotension and bradycardia, however, administration of repeated boluses or an infusion of a narcotic receptor antagonist will be required. In the management of severe hypotension, a pulmonary artery catheter will help guide the need for fluid and inotrope administration, and avoid inappropriate administration of diuretics in patients with NCPO, which requires inten- sive ventilatory support. ${ }^{45}{ }^{47}$ There is very limited evidence on which to base guidelines for the treatment of arrhythmias caused by narcotic overdose. All patients should be investigated, and hypoxic, metabolic, and electrolyte deficits corrected. Haemodynamically unstable tachyarrhythmias require urgent cardioversion. As the misused drug is rapidly metabolised, the majority of arrhythmias are short lived; thus it is preferable to avoid the use of antiarrhythmic agents where possible, to minimise the risk of proarrhythmic interactions. If treatment is needed for the management of supraventricular tachyarrhythmias, conventional agents such as adenosine, $\beta$ blockers, verapamil, and digoxin have been recommended $^{48}$; however, experience with their use is limited. There are no useful published data to guide selection of antiarrhythmic agents in the management of ventricular tachyarrhythmias, which should therefore be managed along conventional lines. Persistent bradycardia may require atropine or temporary cardiac pacing. The patient should be closely monitored for a prolonged period $\left(8-24\right.$ hours $\left.^{45}{ }^{47}\right)$, as NCPO can occur late.

\section{Volatile substance misuse}

The recreational use of inhaled volatile substances to achieve a state of intoxication is an increasingly common problem, reported by $3.5-5 \%$ of adolescents. ${ }^{49}$ Misuse of volatile substances is most common in young males, who account for $90 \%$ of all fatalities. ${ }^{50}$ The products commonly used are legal, cheap, and easily available. Children and adolescents often employ deep breathing techniques with the volatile substances contained in a plastic bag or bottle, a crisp packet, or a soaked handkerchief, to maximise the inhaled concentration of the active agent. Some misusers may spray the compound directly into the oral cavity. Most volatile substances can be detected in blood if samples are obtained within 10 hours of exposure. Urinary analysis for metabolites may extend the time during which exposure can be detected, but only for a limited number of volatile substances such as toluene, tylene, and chlorinated solvents. ${ }^{51}$

Following inhalation of volatile substances, the desired effects of euphoria, excitement, and a feeling of invulnerability occur rapidly. ${ }^{51} \mathrm{~A}$ wide variety of detrimental cardiovascular effects can be induced in an unpredictable fashion, and these are responsible for the majority of sudden deaths. ${ }^{51-53}$ Some volatile substances may induce potentially lethal tachyarrhythmias by sympathetic activation or by increasing myocardial sensitivity to circulating catecholamines..$^{51-54}$ The exact mechanism underlying this sensitisation remains unclear, but animal studies suggest that it may be related to changes in cellular membrane function and transmembrane ionic conductance. $.^{56} \mathrm{Hy}-$ poxia is common with volatile substance misuse and is related to respiratory depression, aspiration, the placement of bags over the head and neck, the formation of carboxyhaemoglobin and methaemoglobin, or laryngeal 
Table 1 Summary of the cardiovascular effects of the commonly misused non-prescription recreational drugs, and therapeutic guidelines

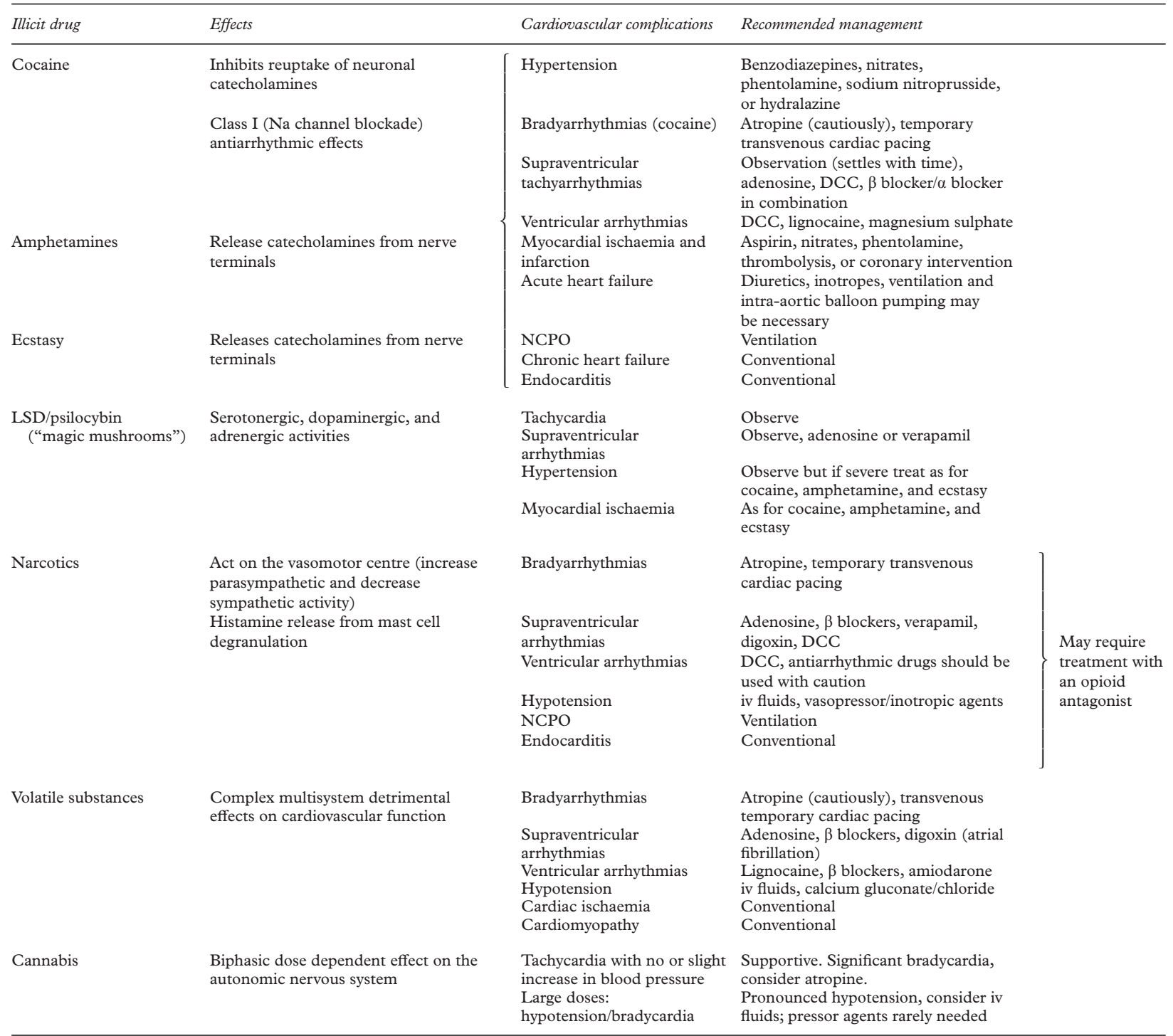

General measures: ensure adequate airway, breathing, circulation, correct any hypoxic, metabolic, and electrolyte disturbances

DCC, DC cardioversion; NCPO, non-cardiogenic pulmonary oedema.

oedema and spasm. ${ }^{49} 5052$ Hypoxia may combine with electrolyte disturbances induced by volatile substance misuse to facilitate the induction of potentially lethal tachyarrhythmias. $^{5157}$

In some individuals, volatile substances may induce lethal bradyarrhythmias. In animal studies some volatile compounds act directly on the heart to reduce sinoatrial node automaticity, prolong the PR interval, and induce atrioventricular block. ${ }^{58}$ Volatile substances sprayed directly in the oral cavity can result in intense vagal stimulation. ${ }^{53}$ Profound bradycardia induced by one or both of these mechanisms can evolve into asystole or secondary ventricular tachyarrhythmias. Some volatile substances are structurally related to the agents used in general anaesthesia, and it is therefore not surprising that myocardial depression and hypotension can be induced. ${ }^{59}$ In some instances, myocardial ischaemia and infarction can be produced by coronary vasospasm, a reduction in the oxygen carrying capacity of the blood related to the formation of carboxyhaemoglobin or methaemoglobinaemia, or excessive sympathetic stimulation..$^{59-63}$ Chronic misuse of some volatile substances can induce a poorly characterised cardiomyopathy. ${ }^{60} 64$

Patients who present with cardiovascular complications related to volatile substance misuse should be managed in a calm nonthreatening environment, with sedation if necessary. Hypoxia and chemical disturbances should be corrected to optimise myocardial electrical stability. Haemodynamically unstable tachyarrhythmias require prompt DC cardioversion. Profound bradyarrhythmias may be treated cautiously with atropine or temporary cardiac pacing. Hypotension can be treated with intravenous fluids, guided by a pulmonary artery catheter if necessary. Inotropic agents are best avoided if possible, as they may induce refractory ventricular tachyarrhythmias in the electrically unstable myocardium. Calcium administration may help to reverse myocardial depression. ${ }^{65}$ In patients with sustained tach- 
yarrhythmias, $\beta$ blockers or amiodarone may help to combat sympathetic activation and are the antiarrhythmic drugs of choice. ${ }^{59}{ }^{66}$ Cardiac ischaemia should be managed with oxygen, vasodilators, and reperfusion treatment if necessary.

\section{Cannabis}

Cannabis is commonly smoked and is rapidly absorbed through the lungs. Absorption is slower and less predictable after the less common practice of gastrointestinal ingestion. The physiological effects of the drug are apparent shortly after absorption, persisting for four to six hours unless administration is repeated. Cannabis has a plasma half life of $20-30$ hours $^{67}$ and can be detected in the urine for several days in occasional users, and for up to two months in heavy users. Cannabis has a biphasic effect on the autonomic nervous system. ${ }^{67-70}$ At low or moderate doses the drug leads to an increase in sympathetic activity and a reduction in parasympathetic activity, producing a tachycardia and an increase in cardiac output. At high doses, sympathetic activity is inhibited and parasympathetic activity increased, leading to bradycardia and hypotension. Reversible ECG abnormalities affecting the $\mathrm{P}$ and $\mathrm{T}$ waves and the ST segment have been reported, although it is not clear whether these changes are related to drug ingestion independently of effects on heart rate. ${ }^{71}$ An increase in supraventricular and ventricular ectopic activity occurs, but life threatening tachy- or bradyarrhythmias have not been reported. In patients with ischaemic heart disease, cannabis increases the frequency of anginal symptoms at low levels of exercise, owing to an increase in myocardial oxygen demand resulting from the drug induced increase in heart rate and myocardial contractility. ${ }^{72}$

The clinical features of acute intoxication may be subtle, and are largely related to the well described central nervous system effects of the drug. In the absence of major underlying structural heart disease, the autonomically mediated changes in heart rate and blood pressure are usually well tolerated. Where necessary, hypotension usually responds to intravenous fluid administration. Serious arrhythmias and major cardiovascular collapse are rare, but the combined use of cannabis and other recreational drugs (particularly cocaine, amphetamine, and ecstasy) may lead to synergistic detrimental effects on the autonomic nervous system or on plasma drug concentrations.

\section{Conclusions}

Various non-prescription illegal recreational drugs are regularly misused on a large scale by adults and adolescents in the UK. In addition, there are increasing reports of inadvertent, accidental ingestion of illegal drugs by children. ${ }^{73}$ All these agents can induce major acute changes in cardiovascular function, and may also cause irreversible damage to the heart. Some individuals with an established pattern of recreational drug misuse may present after the simultaneous administration of intravenous cocaine or amphetamines and heroin ("speedballs") on a background of the combined use of alcohol and cannabis. Adolescents frequently take volatile substances, alcohol, and cannabis at the same time. These combinations of recreational drugs may produce synergistic detrimental effects on cardiovascular function. Current UK estimates suggest that approximately 1800 deaths a year are related to some form of illegal drug misuse, and morbidity levels are much higher.

Many patients who present with complications will be unable or unwilling to provide a history of illegal drug ingestion, but it should always be suspected and looked for in those presenting with unexplained or unusual cardiovascular disturbances, particularly when these are associated with central nervous system dysfunction. The key to successful management of the cardiovascular complications of drug misuse is a high index of suspicion, leading to early recognition and appropriate intervention (table 1). Urine toxicology may help to establish a retrospective diagnosis. There are no prospective, randomised, placebo controlled human studies evaluating the best treatment options, and the therapeutic guidelines provided in this article are based on case reports, retrospective analyses, and animal studies. Once patients are deemed medically fit, the supervising cardiologist should ensure that they are referred for further rehabilitation and psychiatric assessment. With the increasing frequency of drug misuse and its extension into a wider range of age and social groups, cardiologists need to be alert to the detrimental and life threatening cardiovascular effects associated with the use of these common compounds.

We gratefully acknowledge the advice provided by Dr John Ramsay, Toxicology Unit, St George's Hospital Medical School, during the preparation of the manuscript.

1 Chiueh C, Kopin J. Centrally mediated release of cocaine by endogenous epinephrine and norepinephrine from the sympathoadrenal medullary system of unanesthetised rats. f Pharmacol Exp Ther 1978;205:148-54.

2 Mouhaffet A, Madu E, Satmary W, et al. Cardiovascular complications of cocaine. Chest 1995;107:1426-34.

3 Burke WM, Ravi NV. Urinary excretion of cocaine. Ann Intern Med 1990;112:548-9.
Murke

4 Henning RJ, Wilson RD, Glauser JM. Cocaine plus ethanol is more cardiotoxic than cocaine or ethanol alone. Crit Care Med 1994;22:1896-906.

5 Lukas SE, Sholar M, Kouri E, et al. Marihuana smoking increases plasma cocaine levels and subjective reports of 1994;48:715-21.

6 Albertson T, Walby W, Derlet R. Stimulant induced pulmonary toxicity. Chest 1995;198:1140-9.

7 Mendelson J, Jones RT, Upton R, et al. Methamphetamine and ethanol interaction in man. Clin Pharmacol Ther 1995; 57:559-68.

8 Henry JA, Jeffreys KJ, Dawling S. Toxicity and death from 3,4-methylenedioxymethamphetamine ("ectasy"). Lancet 1992;340:384-7.

9 Smit A, Wieling W, Voogel A, et al. Orthostatic hypotension due to suppression of vasomotor flow after amphetamine intoxication. Mayo Clin Proc 1996;71:1067-70.

10 Smith H, Liberman HA, Broody SL, et al. Acute myocardial infarction temporally related to cocaine use. Ann Intern Med 1987;107:13-18.

11 Mittleman MA, Mintzer D, Maclure M. Triggering of myocardial infarction by cocaine. Circulation 1999;99:2737-41.

2 Choi YS, Pearl WR. Cardiovascular effects of adolescent drug abuse. F Adolesc Drug Abuse 1989;10:332-7.

13 Bashour T. Acute myocardial infarction resulting from amphetamine abuse: spasm thrombus interplay? Am Heart f 1994;128:1237-8.

14 Derlet RW, Rice P, Horowitz BZ, et al. Amphetamine toxicity: experience with 127 cases. F Emerg Med 1989;7: 157-61. 
15 Furst S, Fallon S, Reznik G, et al. Myocardial infarction after inhalation of methamphetamine. N Engl f Med 1990 323:1147-8.

16 Tonga G, Tempesta E. Platelet responsiveness and biosynthesis of thrombaxane and prostacyclin in response to in vitro cocaine treatment. Homeostasis 1985;15:100-7.

17 Joffe BD, Broderick TM, Leier CV. Cocaine induced coronary artery dissection. $N$ Engl f Med 1994;330:510-11.

18 Citron $\mathrm{B}$, Halperm $\mathrm{M}$, McCarron $\mathrm{M}$, et al. Necrotising angiitis associated with drug abuse. $N$ Engl $7 \mathrm{Med}$ 1970;283:1003-11

19 Weiner R, Lockhart JT, Schwartz RG. Dilated cardiomyopathy and cocaine abuse: report of two cases. $A m \mathcal{F} \mathrm{Med}$ 1986;81:699-701.

20 Milroy CM, Clark JC, Forrest ARW. Pathology of deaths associated with "ecstasy" and "eve" misuse. F Clin Pathol 1996;49:149-53.

21 Hong R, Matsuyama E. Cardiomyopathy associated with the smoking of crystal methamphetamine. $7 A M A 1991$; 265:1152-4.

22 Davis GG, Swalwell CI. Acute aortic dissection and ruptured berry aneurysms associated with methamphetamine abuse. F Forensic Med 1994;39:1481-5.

23 Isner JM, Estes M, Thompson PD. Acute cardiac events temporally related to cardiac abuse. $N$ Engl $\mathcal{F} \mathrm{Med}$ 1986;315:1438-43.

$24 \mathrm{Om} \mathrm{A}$, Ellenbogen KA, Vetrovec GW. Cocaine-induced bradyarrhythmias. Am Heart f 1992;124:232-4.

25 Hollander J. The management of cocaine associated myocardial ischemia. N Eng f Med 1995;333:1267-71.

26 Gay GR, Loper KA. The use of labetalol in the managemen of cocaine crisis. Ann Emerg Med 1988:17:282-3.

27 Billman GE. Effect of calcium channel antagonist on cocaine-induced malignant arrhythmias: protection against ventricular fibrillation. 7 Pharmacol Exp Ther 1993;266. 407-16.

28 Negus BH, Willard JE, Hillis LD. Alleviation of cocaineinduced coronary vasoconstriction with intravenous verainduced coronary vasoconstriction with

29 Derlet RW, Tseng CC, Albertson TE. Cocaine toxicity and the calcium channel blockers nifedipine and nimodepine in the calcium channel blockers nifed

30 Derlet RW, Albertson TE. Potentiation of cocaine toxicity with calcium channel blockers. Am f Emerg Med 1989;7 $464-8$

31 Hollander J, Burstein J, Hoffman R, et al. Cocaine associated myocardial infarction. Clinical safety of thrombolytic therapy. Ann Emerg Med 1995;26:702-6.

32 Hollander J, Hoffman R, Burstein J, et al. Cocaine associated myocardial infarction. mortality and complications. Arch Intern Med 1995;155:1081-6.

33 Shih R, Hollander J, Burstein J, et al. Clinical safety of lidocaine in patients with cocaine associated myocardial infarction. Ann Emerg Med 1995;26:702-6.

34 Beckman KJ, Parker RB, Hariman RJ, et al. Hemodynamic and electrophysiological actions of cocaine: effects of sodium bicarbonate as an antidote in dogs. Circulation 1991;83:1799-807.

35 Gary N, Saidi P. Methamphetamine intoxication: a speedy new treatment. Am F Med 1978;64:537-40.

36 Burkhalter A, Julius DJ, Katzung BG. Histamine, serotonin and the ergot alkaloids. In: Katzung BG, ed. Basic and clinical pharmacology, 7th ed. Stamford, Connecticut: Appleton and Lange, 1998:279.

37 Leikin JB, Krantz AJ, Zell-Kanter M, et al. Clinical features and management of intoxication due to hallucinogenic drugs. Med Toxicol Adverse Drug Exp 1989;4:324-50.

38 Abraham HD, Aldridge AM. Adverse consequences of lysergic acid diethylamide. Addiction 1993;88:1327-34

39 Klock JC, Boerner U, Becker CE. Coma, hyperthermia and bleeding associated with massive LSD overdose: a report of eight cases. West F Med 1974;120:183-8.

40 Borowiak KS, Ciechanowski K, Waloszczyk P. Psilocybin mushroom (Psilocybe semlanceata) intoxication with myocardial infarction. Clinical Toxicol 1998;36:47-9.

41 Peden NR, Bissett AF, Macaulay KEC, et al. Clinical toxicology of "magic mushroom" ingestion. Postgrad Med $\mathscr{f}$ 1981;57:543-5.

42 Lipski J, Stimmel B, Donoso E. The effect of heroin and multiple drug abuse on the ECG. Am Heart $\mathcal{F} 1973 ; 86$ : 663-8.
43 Holland DR, Steinberg MI. Electrophysiologic properties of propoxyphene and norpropoxyphene in canine cardiac onducting tissues in vitro and in vivo. Toxicol $A p p l$ Pharmacol 1979;47:123-33.

44 Remskar M, Noc M, Leskovsek B, et al. Profound circulatory shock following heroin overdose. Resuscitation 1998;38:51-3

45 Osterwalder JJ. Patients intoxicated with heroin or heroin mixtures: how long should they be monitored? Eur f Emerg Med 1995;2:97-101.

46 Duberstein J, Kaufman DF. A clinical study of an epidemic of heroin intoxication and heroin induced pulmonary oedema. Am f Med 1971;51:704-14.

47 Wang ML, Lin JL, Bullard MJ. Heroin lung: a report of two cases. F Formosan Med Assoc 1994;93:170-2.

48 Benowitz NL, Goldschlager N. Cardiac disturbances. In: Haddard LM, Shannon MW, Winchester JF, eds. The clinical management of poisoning and drug overdose, 3rd ed. Philadelphia: WB Saunders, 1998:115.

49 Langa A. Volatile substance abuse: a brief report. $\mathrm{Br}$ f Clin Pharmacol 1993;47:94-6.

50 Ashton CH. Solvent abuse. BMF 1990;300:135-6.

51 Meredith TJ, Ruprah M, Liddle A, et al. Diagnosis and treatment of acute poisoning with volatile substances. Hum Toxicol 1989;8:277-86.

52 Flanagan RJ, Ives RJ. Volatile substance abuse. Bull Narcotics 1994;46:49-78.

53 Shepherd RT. Mechanism of sudden death associated with volatile substance abuse. Hum Toxicol 1989;8:287-92.

54 Williams DR, Cole SJ. Ventricular fibrillation following butane gas inhalation. Resuscitation 1998;37:43-5.

55 Kaufman JD, Silverstein MA, Moure-Eraso R. Atrial fibrillation and sudden death related to occupational solvent exposure. Am f Indust Med 1994;25:731-5.

56 Lessard Y, Paulet G. A proposed mechanism for cardiac sensitisation: electrophysiological study of effects of difluorodichloromethane and adrenaline on different types of cardiac preparations isolated from sheep hearts. Cardiovasc Res 1986;20:807-15.

57 Waldron HA. Effects of organic solvents. Br $\mathcal{F}$ Hosp Med 1981;26:645-9

58 Taylor GJ, Harris WS. Glue sniffing causes heart block in mice. Science 1970;170:866-8.

59 Cunningham SR, Dalzell GWN, McGirr P, et al. Myocardial infarction and primary ventricular fibrillation after glue sniffing. BMF 1987;294:739-40.

60 Hussain TF, Heidenreich PA, Benowitz N. Recurrent non-Q-wave myocardial infarction associated with toulene abuse. Am Heart $\mathcal{F}$ 1996;131:615-16.

61 Wodka RW, Jeong EWS. Cardiac effects of inhaled typewriter correction fluid. Ann Intern Med 1989;110:91.

62 Gunn J, Wilson J, Macintosh AF. Butane sniffing causing ventricular fibrillation. Lancet 1989;i:617.

63 Stewart RD, Hake CL. Paint-remover hazard. FAMA 1976; 235:398-41.

64 Wiseman MN, Banim Seamus B. "Glue sniffer's" heart? $B M F$ 1987;294:739.

65 Herd PA, Lipsky M, Martin HF. Cardiovascular Effects of 1,1,1-tri-chloroethane. Arch Environ Health 1974;28:22733

66 Adgey AAJ, Jonston PW, McMechan S. Sudden cardiac death and substance abuse. Resuscitation 1995;29:219-21.

67 Olson KR, ed. Poisoning and drug overdose, 3rd ed. Stamford, Connecticut: Appleton and Lange 1999.

68 Rinaldi L. Marijuana: a research overview. Alaska Med 1994;36:107-13.

69 Tashkin D. Cannabis. Ann Intern Med 1978;89:539-49.

70 Kanakis C, Pouget C, Rosen KM. The effects of delta 9 tetrahydrocannabinol on cardiac performance with and without beta blockade. Circulation 1976;53:703-7.

71 Kochar M, Hosko MJ. Electrocardiographic effects of marijuana. $7 A M A$ 1973;225:25-7.

72 Shapiro BJ, Reiss S, Sullivan SF, et al. Cardiopulmonary effects of marijuana smoking during exercise. Chest 1976;70:441.

73 Beatie J. Children poisoned with illegal drugs in Glasgow. BMF 1999;318:1137. 